\title{
Comment est née la « Revue française de gestion "
}

La Fondation nationale pour l'enseignement de la gestion des entreprises est fondée en... mai 1968. Le premier numéro de la Revue française de gestion est publié au printemps 1975. Entre les deux dates, une guerre des Anciens et des Modernes secoue le monde de l'entreprise. Et l'enseignement de la gestion accomplit sa révolution culturelle. Quarante plus tard, retour sur les circonstances de la naissance de la première revue française des sciences de l'entreprise, des organisations et de l'action collective. 


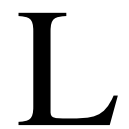

oi des cycles ou simple coïncidence ? Quarante ans après la publication du premier numéro de la « Revue française de gestion », la France fait face à des défis de même nature que ceux du milieu des années 1970. Hier comme aujourd'hui, le débat public s'articulait autour d'une poignée de dominantes : 1) l'ordre du monde occidental est bousculé par la montée en puissance de nouveaux acteurs ; 2) des innovations de rupture dynamitent les anciens systèmes de production et d'échange ; 3) le chômage inonde des pans entiers de territoires et de métiers ; 4) l'école et l'université ont du mal à arbitrer entre l'approfondissement « vertical » des disciplines et l'hybridation, la pluridisciplinarité, leviers des processus d'innovation en période instable. Dans le prolongement des événements de mai 1968, le débat public se focalisait déjà sur le thème du « monde d'après » comme s'il était acquis qu'un nouveau modèle de croissance - voire de civilisation - allait prendre le relais du modèle ancien. Même discours quarante ans plus tard.

Le premier numéro de la « Revue française de gestion » est mis en vente au printemps 1975. Boulevard Haussmann, une équipe dédiée fonctionne depuis l'année précédente dans les locaux de la Fondation nationale pour l'enseignement de la gestion des entreprises (FNEGE). Dès l'automne 1974, un numéro zéro - destiné à devenir le numéro 1 - a été testé auprès des «trois mondes » en compétition dans le domaine de l'enseignement de gestion français : le monde universitaire, attentif au respect des critères académiques ; le monde consulaire, désireux de maintenir son leadership dans les formations commerciales; le monde patronal, soucieux de préparer la relève de la classe dirigeante de l'après-guerre. Pour Philippe Agid, alors secrétaire général de la FNEGE, la décision ultime de lancement ne peut se faire qu'au prix d'un accord général sur le projet éditorial de la revue et d'un engagement des responsables des trois réseaux à devenir fournisseurs de contenus. Le souci de neutralité au regard des trois familles explique que la FNEGE ait recruté un journaliste professionnel issu de la presse économique - l'auteur de ces lignes - pour assurer la rédaction en chef de la revue. Il est entouré de deux adjoints : Michel Bernard, permanent de la Fondation et Bernard Pras, tout jeune professeur de l'ESSEC qui va jouer un rôle clé tout au long des décennies à venir. Un comité de rédaction est constitué en vue d'assurer l'enracinement institutionnel de la revue. Il cédera rapidement la place à un comité d'orientation plus conforme aux exigences d'une revue scientifique mais son rôle est utile tout au long de la période de démarrage. Il est composé à parité de dirigeants d'entreprise (parmi lesquels Pierre Bellon de Sodexho, Marcel Boiteux d'EDF, Octave Gélinier de la CEGOS, Bernard Hanon de Renault et Jacques Lesourne de la SEMA) et de représentants du monde de l'enseignement de gestion (parmi lesquels Maurice Saias de l'université Aix-Marseille, Jean-Claude Tarondeau de l'ESSEC, Alain Bienaymé et Alain Cotta de Paris IX Dauphine et Christian Vulliez d'HEC).

Le lancement de la revue est précédé d'une restructuration en profondeur, sous l'égide de la FNEGE, du secteur de l'édition spécialisée dans les sciences de gestion. C'est la première mission du rédacteur en chef de la revue qui va naître. Depuis la parution du Défi Américain de Jean-Jacques Servan-Schreiber en 1967, plusieurs revues 
généralistes de management ont vu le jour, tentant sans succès de concilier des contenus de niveau variable avec une exploitation sans subvention. Le groupe Express, qui publie sous la direction d'Henri Dougier Le Management à l'ombre de son magazine à succès L'Expansion, renonce après quelques années d'exploitation déficitaire. À l'exception d'une revue publiée sous le titre Direction et Gestion, tous les éditeurs répondent aux propositions de la FNEGE. Jean-Baptiste Eggens, rédacteur en chef de la revue Direction devient conseiller de la RFG. De son côté, la CEGOS ne se contente pas d'interrompre la publication de sa revue Hommes et Techniques, elle apporte à la revue un important fichier d'abonnés, ce qui donnera dès le départ un solide coup de pouce à sa diffusion.

\section{QUADRATURE DU CERCLE}

Une fois le paysage concurrentiel éclairci et les conditions d'exploitation définies, l'équipe de la rédaction se prépare au plus difficile : définir la ligne éditoriale de la nouvelle revue. Quel projet pour quel public ? On devine la difficulté qu'il y a à associer le monde académique et le monde des entreprises dans le lancement d'une revue que les uns souhaitent orientée vers la recherche et les autres, vers l'aide à la pratique du management - stratégie et organisation. Où placer le curseur? Comment retenir l'attention des boursiers de la FNEGE revenus des États-Unis avec un $\mathrm{PhD}$ et voués à former le noyau dur des corps professoraux permanents de l'enseignement de gestion? Comment devenir une plate-forme d'accueil pour les écrits des candidats à l'agrégation de sciences de gestion qui se profile à l'horizon ?
Comment attirer dans une publication à vocation généraliste des auteurs soucieux d'être reconnus dans le cercle étroit des spécialistes de leur discipline ? Comment réussir à créer une revue qui soit à la fois respectueuse des critères académiques et assez « lisible» pour franchir le mur des entreprises et des administrations ? Une revue capable de capter l'attention de la nouvelle élite de la finance, du marketing, des ressources humaines ou de la stratégie d'entreprise, la génération frondeuse du baby boom formée à HEC, l'ESSEC, Dauphine ou Sup de Co Lyon qui commence à secouer les colonnes du temple dans les grands ensembles industriels et financiers issus de l'ère Pompidou...

Mission impossible ? L'équipe fondatrice sait qu'il lui faudra procéder en deux temps. D'abord se faire un nom, exister au sein de la population mixte des enseignants, des dirigeants d'entreprise et des cadres de service public. Puis dans un second temps, resserrer l'angle d'attaque des publics et des contenus. La crédibilité future de la revue dépendra de la qualité de ses procédures de sélection d'auteurs et d'articles. Mais la légitimité ne se conquiert pas par décret. Une période d'apprentissage et de mise à l'épreuve est nécessaire avant la reconnaissance par une communauté de chercheurs ou de praticiens de haut niveau.

La démarche pragmatique des conseillers de la FNEGE et de la RFG est guidée par une intuition : compte tenu des bouleversements économiques et sociaux qui déstabilisent le pays, il importe de ne pas enfermer la jeune revue dans un ghetto de recherche désincarnée. Avant d'offrir aux enseignants de gestion le support de publication qui servira leur carrière, il convient de s'assurer de leur complète immersion dans le contexte social 
et entrepreneurial du moment. Voire, de les encourager à apporter leur contribution au débat national qui s'engage à la suite du premier choc pétrolier.

\section{LE MONDE DE L'ENTREPRISE EN FUSION}

Il faut se replacer dans le contexte de l'époque. En juillet 1974, un mois à peine après son installation à l'Elysée, Valéry Giscard d'Estaing confie à Pierre Sudreau la présidence d'un comité chargé de proposer une réforme de l'entreprise. Quelques mois plus tard, 200000 Français achèteront l'édition en livre de poche du « Rapport Sudreau ». Au même moment, Yvon Gattaz le patron d'une ETI - Radiall - lance le mouvement Ethic pour défendre la cause des entreprises à «taille humaine ». Le grand patronat n'est pas en reste. Sous l'impulsion de François Ceyrac (CNPF), Jean Chenevier (pétrole BP) et François Dalle (L'Oréal), l'Institut de l'Entreprise est créé en 1975 dans le but d'associer les grandes entreprises à la résolution des problèmes de l'État, de l'école et de la société.

Au lendemain des événements de 1968 - le décret qui fonde la FNEGE date de mai 1968 ! - le monde de l'entreprise est en ébullition. La première grande OPA - celle de BSN sur Saint Gobain - et la fronde des salariés de Lip déclenchent de vives controverses entre patrons progressistes et conservateurs. En juillet 1972 aux Assises du CNPF à Marseille, Antoine Riboud, qui vient de marier BSN et Gervais Danone, fait scandale avec un discours fracassant sur les archaïsmes du modèle social français. Face à lui, Ambroise Roux, PDG de la Compagnie générale d'électricité - le conglomérat Alcatel-Alstom totalement désintégré quarante ans plus tard - se pose en gardien de la tradition face aux tentations " gauchistes » de mouvements du type « Entreprise et Progrès ». Tradition qui lui fait préférer les méthodes de la CGT à celles de la CFDT et le capitalisme d'économie mixte au libéralisme à l'anglo-saxonne.

\section{LA GESTION MONTE EN GAMME}

Au milieu des années 1970, la FNEGE n'est pas à l'écart du climat orageux qui règne dans le monde patronal français. Placée de manière constitutive au croisement de la sphère publique et de la sphère privée, elle bénéficie du soutien actif, côté dirigeants d'entreprise, de réformateurs modérés à l'image de Jean-Yves Eichenberger, PDG de Penarroya qui se trouve être le président de la FNEGE au moment du lancement de la revue. De son côté, le groupe Lafarge, à travers ses présidents successifs Marcel Demonque et Olivier Lecerf, est perçu comme le chef de file d'un courant patronal qui voit dans la montée en gamme de l'enseignement de gestion le préalable indispensable à toute réforme du modèle français de management.

Cette montée en gamme, les trois réseaux d'enseignement de gestion y travaillent alors activement comme en témoignent la montée en puissance des IAE, le succès immédiat de l'expérience de ParisDauphine ou l'élan créé par la loi sur la formation permanente.

\section{PARRAINAGE MÉDITERRANÉEN}

Tel est le paysage à la veille de la publication du premier numéro de la Revue française de gestion. Dans les semaines qui précèdent, l'équipe de rédaction en chef 
mène son enquête sur les sources d'inspiration communes aux chefs d'entreprise, aux hauts fonctionnaires et aux responsables d'établissement qui gravitent autour de la FNEGE. La surprise est grande de voir que tous sans exception invoquent une même filiation intellectuelle. Deux hommes de la Méditerranée symbolisent à leurs yeux le lien organique entre l'école, l'université, l'entreprise et la société. Le premier est Gaston Berger, dont l'influence reste profonde en 1975 alors qu'il a disparu quinze ans plus tôt dans un accident de voiture. Le second, considéré comme son disciple, est Pierre Tabatoni, premier secrétaire général de la FNEGE.

Gaston Berger était tout à la fois philosophe et chef d'entreprise. Il dirigeait une entreprise d'engrais à Marseille tout en se vouant à l'art - il était le père du chorégraphe Maurice Béjart - et à l'enseignement de la philo. Partageant sa vie entre deux mondes qui s'ignorent, sa carrière bascule quand il est nommé directeur général de l'enseignement supérieur au ministère de l'Éducation. Il devient alors le véritable « inventeur » des études de gestion en France dans les années 1950.

Gaston Berger, fût le fondateur, à travers les IAE, du «CAAE » (Certificat d'aptitude à l'administration des entreprises, en 1954, ancêtre français des DESS et d'une certaine façon des MBA puisque désireux de donner une « double » compétence à des diplômés d'écoles d'ingénieurs, des titulaires d'une maîtrise de sciences éco, droit, sciences ou lettres). Le CAAE devenu MAE dans bien des cas est le point commun des IAE même si ceux-ci ont aujourd'hui des configurations très différentes

De son côté, Pierre Tabatoni avait été formé des deux côtés du pont - réel et symbolique - qui relie le département d'économie et la business school d'Harvard. D'où une double culture d'économiste et d'enseignant de gestion qui lui assurera une légitimité dans des univers en concurrence et qui le rendra constamment ouvert aux approches pluridisciplinaires. Il est tout à la fois le fondateur de l'IAE d'Aix en Provence, de l'université Paris-Dauphine et il jouera plus tard un rôle actif dans l'avènement de l'agrégation de Sciences de gestion. La boucle est bouclée. Au printemps 1975, la Revue française de gestion vient tout naturellement ajouter sa brique aux dispositifs initiés par les deux grands réformateurs de l'enseignement de gestion français. Le premier numéro de la revue paraît au moment où s'affirme définitivement en France l'autonomie des disciplines qui traitent des processus de décision et d'organisation. Dix articles, cent cinquante pages. Entre autres, un article sur la théorie générale de la firme, une analyse sur les conflits dans les canaux de distribution, un recensement des recherches en gestion terminées en 1974 et un entretien avec le directeur financier du groupe Total. Dès novembre 1975, la revue publie son deuxième numéro. Une institution pérenne s'installe dans le paysage. 
